\title{
On understanding the transition from internal to external flash boiling
}

\author{
Songzhi Yang ${ }^{1}$, Yanfei Li ${ }^{* 2}$, Jingyu Zhang ${ }^{2}$, Zijie Zhao ${ }^{2}$, Shijin Shuai ${ }^{1,2}$ \\ ${ }^{1}$ Institute for Aero Engine, Tsinghua University, Beijing, China \\ ${ }^{2}$ State Key Laboratory of Automotive Safety and Energy, Tsinghua University, Beijing, China \\ ${ }^{*}$ Corresponding author email: liyf2018@tsinghua.edu.cn
}

\begin{abstract}
Flash boiling has been widely investigated experimentally and numerically in the world for its non-negligible influences on air-fuel mixing, combustion and emission in gasoline engines. Nevertheless, the internal and external flash boiling, and the transition regime therein are still far fully understood. The present work is aimed to shed some light on this issue through numerical simulation approach. The simulation tool is based on homogeneous relaxation (HRM) model implemented in CONVERGE CFD solver. Through a series of case-sensitive simulations for hexane fuels with varied ambient pressure, the flashing scheme has transferred from the external flash state to internal flash state. By redefining the pressure ratio $\left(R p_{d}\right)$ based on the ratio of the saturation pressure at real-time temperature and real-time pressure, the phase change regions or the superheated aeras in the spray are clearly presented. It is found that the superheated or potential phase change regions have shifted gradually from the nozzle inside in the upper stream to the downstream spray during the transition of non-flashing state, external flash boiling to fierce internal flash boiling regime. Moreover, the timing for the transition of internal flash boiling and external flash boiling may correspond to the disappearance of Mach disk. The current research is expected to provide more insights to the understanding of the internal and external flash boiling.
\end{abstract}

\section{Keywords:}

Internal flash boiling, external flash boiling, real time Rp

\section{Introduction}

The flash boiling phenomenon appears as the liquid is discharged into the environment with the pressure lower than the saturation pressure of the fuel which has been proven favorable in improving fuel atomization, air-fuel mixing and further combustion in conventional internal combustion engines and scramjet [1], [2]. The study herein mainly focuses on the flash boiling phenomenon in the gasoline direct injection engine namely flash boiling sprays.

Current work is mainly concentrated on the exploration of the internal and external flash boiling. Previous studies about the internal/external flash boiling are mostly based on experimental observations [3]-[5]. The internal/external flash boiling is defined based on the inception position of jet expansion or instantaneous phase transition [3]. In the external flashing regimes, the liquid jet remains intact for a while after exiting from the nozzle before going through instantaneous shattering or expansion. As confirmed by Oza [3] and Guo [5],this category of flashing mechanism is rather difficult to control, requiring a strict control of nozzle geometry (i.e. aspect ratio, inlet roundness, surface roughness, etc.) and the thermal physical properties of fuel. However, this regime is also deemed as a transition stage from no-flash boiling to flash boiling as observed in [4], [6], [7] in which the fuel is generally at a low superheated degree (Figure 1). Other disputes also occur in the understanding of the multiphase flow in external flash boiling. According to Oza [3] and Guo [5], the liquid core in the idle stage is regarded more as single phase metastable flow which differs from the studies in [6], in which the fluid at low superheated state is deemed as multiphase with abundant 
bubbles as ejecting from the nozzle (Figure 1). Until now, no consensus is reached related to the multiphase flow state during the external flash boiling. Contrarily, the internal flashing regime appears mostly as the injected fuel is at a high superheated degree. The fuel has been through fast phase transition or under-expansion inside the nozzle, producing two-phase flow inside the nozzle as shown in Figure 1. The generated two-phase flow instantaneously shatters as exiting from the nozzle without delaying. Yet, this viewpoint that the fluid is already atomized before leaving the nozzle is again challenged by Reitz et al [8]. By using the shortduration backlit photographs instead of the conventional scattered light illumination, they detect the intact liquid core downstream the nozzle exit without evident phase transition. The liquid atomization incepts outside the nozzle exit instead of the internal nozzle.

Moreover, the intense under-expansion behavior outside the nozzle in the internal flash boiling regime is believed to be the main culprit for the spray collapse occurred in the multi-hole injector. However, it is predicted that the external flash boiling can be a potential valid measure to curb spray collapse as mentioned by Guo et al [5].

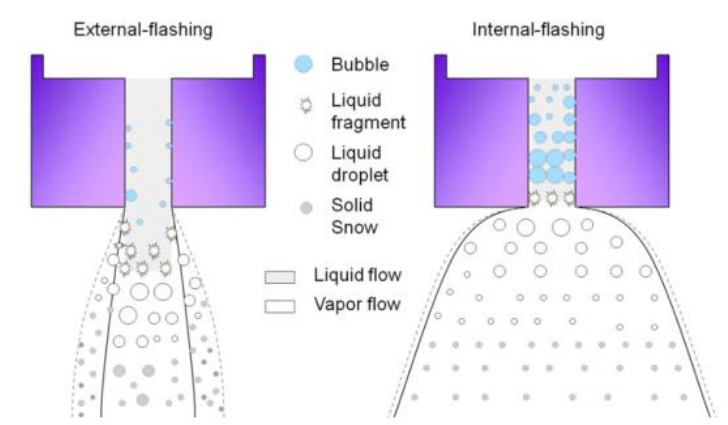

Figure 1 Illustration of external and internal flash boiling, reprinted from ref.[6].

In contrast, the relevant numerical simulations relating to the internal and external flash boiling are extremely scarce. Many previous macroscopic simulations paid more attention to the understanding of mechanism of spray collapses appeared in multiple hole injectors [9]-[11] or the spray penetration, velocity or droplets distribution [12], [13]. Few numerical studies involve comprehensive discussion about the internal and external flash boiling, multiphase flow physics or phase change behavior in the spray. Therefore, current work in this study is aimed to fill in this gap by employing the numerical simulation technique to shed some light on the internal physics especially about the transition mechanism between different schemes and the internal multiphase flow and phase transition characteristics.

The outline of this paper is as follows: Section 2 describes the mathematical models utilized during simulation. Section 3 discusses the simulation setup including the configuration of geometry, mesh discretization strategy and operating conditions of involved test cases. Section 4 presents the validation results between experiments and simulation, and detailed analyses of simulation results. Eventually, Section 5 summaries the conclusion of the current study.

\section{Mathematical models}

The governing equation utilized to solve the flow dynamics is according to the following equation system, namely continuity equation (1), momentum equation (2) and internal energy equation (3). 


$$
\begin{aligned}
& \frac{\partial \rho_{m}}{\partial t}+\frac{\partial \rho_{m} u_{i}}{\partial x_{i}}=\frac{\partial}{\partial x_{j}}\left(\rho D \frac{\partial Y_{m}}{\partial x_{j}}\right)+S_{m} \\
& \frac{\partial \rho u_{i}}{\partial t}+\frac{\partial \rho u_{i} u_{j}}{\partial x_{j}}=-\frac{\partial P}{\partial x_{i}}+\frac{\partial \sigma_{i j}}{\partial x_{j}} \\
& \frac{\partial \rho e}{\partial t}+\frac{\partial \rho e u_{j}}{\partial x_{j}}=-P \frac{\partial u_{j}}{\partial x_{j}}+\sigma_{i j} \frac{\partial u_{i}}{\partial x_{j}}+\frac{\partial}{\partial x_{j}}\left(K \frac{\partial T}{\partial x_{j}}\right)+\frac{\partial}{\partial x_{j}}\left(\rho D \sum_{m} h_{m} \frac{\partial Y_{m}}{\partial x_{j}}\right)
\end{aligned}
$$

In these equations, $\rho, \rho_{m}, Y_{m}$ are the mixture density, species $(m)$ density and species $(m)$ mass fraction respectively, the $\rho_{m}$ can be computed from $\rho$ by using equation (4), $u_{i}$ denotes velocity, $D$ is the mass diffusion coefficient which is the ratio between molecular viscosity $(\mu)$ and Schmidt number $(S c)$, defined as equation (4), $S_{m}$ is the source term accounting for phase change effect, $\sigma_{i j}$ is the viscous stress tensor, defined as equation (5), $P$ is pressure, $e$ is internal energy, $K$ is the conductivity, $h_{m}$ is the species specific enthalpy.

$$
\begin{gathered}
\rho_{m}=Y_{m} \rho, D=\frac{\mu}{s c} \\
\sigma_{i j}=\mu\left(\frac{\partial u_{i}}{\partial x_{j}}+\frac{\partial u_{j}}{\partial x_{i}}\right)+\left(-\frac{2}{3} \mu\right)\left(\frac{\partial u_{k}}{\partial x_{k}} \delta_{i, j}\right)
\end{gathered}
$$

In equation (5), $\mu$ is viscosity, $\delta_{i, j}$ is Kronecker delta.

For the gaseous phase, Redlich-Kwong (RK) equation of state [14] is selected considering it can predict the properties of gas phase well instead of the liquid phase. In contrast, liquid is regarded as compressible, and the density is calculated with the following equation:

$$
\rho=\rho_{\text {ref }} e^{\left(\frac{P-P_{\text {ref }}}{B}\right)}
$$

Where, $\rho_{\text {ref }}$ and $P_{\text {ref }}$ denote the reference density and pressure respectively. $B$ is the bulk modulus.

The computation of liquid properties are based on the linear interpolation from a prepossessing table which includes the general thermodynamic variables (vapor pressure, density, specific heat, etc ) from NIST database[15].

The homogeneous relaxation (HRM) model implemented in CONVERGE CFD software is adopted to resolve the phase change issue. Distinct with the usual phase equilibrium model assuming an infinite phase change rate [16], HRM model has prescribed a finite relaxation time to determine the rate of phase transition.

As confirmed by a list of researchers[17], this model has presented excellent performance in dealing with the classical non-equilibrium physics, such as cavitation and flash boiling.

Specifically, the model is proposed by Bilicki [18]. The rate of evaporation is determined by the following formular:

$$
\frac{D x}{D t}=\frac{\bar{x}-x}{\theta}
$$

$\frac{D x}{D t}$ is the material derivative of $x . x$ represents the instantaneous mass, $\bar{x}$ denotes the equilibrium mass, $\theta$ represents the time scale over which $x$ relaxes to $\bar{x}$. $\theta$ is defined with the following equation for the pressure larger than 10 bar which suits current research: 


$$
\theta=\theta_{0} \alpha^{-0.54}\left(\frac{P_{\text {sat }}-P}{P_{c}-P_{\text {sat }}}\right)^{-1.76}
$$

Where $\theta_{0}=3.84 \mathrm{e}-7 \mathrm{~s}$ is the time constant validated originally for water flow. For current study, it is adjusted to 1e-9 based on experiment measurement. The large value of $\theta$ may cause the inter facial mass transfer to disappear. Extreme small $\theta$ value may lead to the other limit, say homogeneous equilibrium [19]. $\alpha$ is void fraction, $P_{s a t}$ is the saturation pressure. $P_{c}$ is the critical pressure.

In CONVERGE[20], the Volume of Fluid (VOF) method is used to model the two-phase flow. The void fraction $\alpha$ is employed to denote the volume fraction of gas, which is defined as follows.

$$
\left\{\begin{array}{cl}
\alpha=0 & \text { pure liquid } \\
0<\alpha<1 & \text { two-phase }, \alpha=1-\left(1-Y_{\text {gas }}\right) \frac{\rho}{\rho_{\text {liq. }}} \\
\alpha=1 & \text { pure gas }
\end{array}\right.
$$

One noting point is that the void fraction is not solved in the transport equation but calculated directly with the mass fraction and density as shown in equation (9).

For the turbulent model, the generalized RNG $\kappa-\varepsilon$ model was applied. All the simulations are run over a time-resolved transient compressible solver. The time step is constrained by the Courant-Friedrichs-Lewy or CFL condition taking into account the convection, diffusion and Mach effect simultaneously.

\section{Simulation setup}

The simulation configuration is a single-hole injector modified from a commercial five-hole GDI injector. The hole structure is shown in Figure 2. The diameter of the inner hole and counter bore is $0.18 \mathrm{~mm}$ and $0.42 \mathrm{~mm}$ respectively. And the lengths of the inner hole and the counterbore are $0.16 \mathrm{~mm}$ and $0.42 \mathrm{~mm}$, respectively. The base grid size is $0.1 \mathrm{~mm}$. A fixed mesh embedding is adopted for the inner hole and counter bore region with the uniform mesh size of $12.5 \mu \mathrm{m}$. While for the external spray region, Adaptive mesh refinement (AMR) scheme is selected with a refining grid level of 3 which limits the minimal mesh size equaling $12.5 \mu \mathrm{m}$. The total number of grids count 2 million. The computation region is demonstrated as below. The whole computational time for each case lasts $100 \mu$ s to ensure a quasi-stable flow in the near nozzle region. The working fuel is hexane. The injection pressure in all the test cases is set with $10 \mathrm{MPa}$. The injection temperature is fixed at $353 \mathrm{~K}$. The ambient temperature is set with $300 \mathrm{~K}$. As the only variable in the simulation, the ambient pressure is varied from $4 \mathrm{kPa}$ to $500 \mathrm{kPa}$ as shown in Table 1 . The initial superheated degree $R p_{\text {ini }}$. is also presented in the table defined as the ratio of saturation pressure and ambient pressure $\left(\frac{P_{\text {sat }} .(353 K)}{P_{\text {amb. }}}\right)$. The ambient is initially filled with nitrogen.
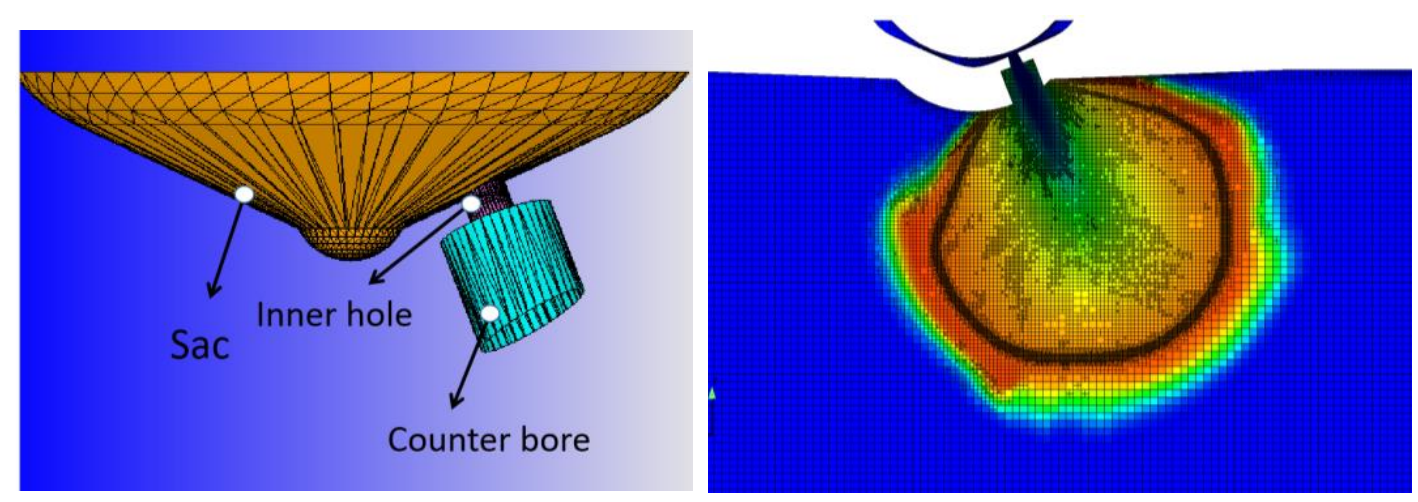
Figure 2 Illustration of geometry configuration and AMR mesh distribution

Table 1 Initial simulation conditions

\begin{tabular}{cccccccccccccc}
\hline$P_{\text {amb. }} / \mathrm{kPa}$ & 4 & 6 & 8 & 10 & 20 & 40 & 60 & 80 & 101 & 200 & 300 & 500 \\
\hline$R p_{\text {ini. }}$ & 36.25 & 24.16 & 18.13 & 14.51 & 7.25 & 3.63 & 2.42 & 1.81 & 1.43 & 0.73 & 0.48 & 0.29 \\
\hline$P_{\text {inj. }}=10 \mathrm{MPa}, T_{\text {inj. }}=353 \mathrm{~K}, T_{\text {amb. }}=300 \mathrm{~K}$ & & & & &
\end{tabular}

\section{Results discussions}

\subsection{Experimental validation}

To validate the efficacy of the simulation model, the simulation results are compared to experimental observations as demonstrated in following Figure 3. The experimental results are obtained at the same operating condition as simulation. The simulation results illustrate the mass fraction distribution of the liquid hexane $\left(Y_{C 6 H_{14} L}\right)$ at the time instant of $100 \mu \mathrm{s}$. With the gradual increment of ambient pressure from $4 \mathrm{kPa}$ to $200 \mathrm{kPa}$, the spray regimes have been through a transition from flash boiling, intermediate boiling to final non-flashing stage. The low ambient pressure can not only facilitate intensive evaporation, but also promote faster expansion of the upper formed bubbles or nuclei which is predicted from the continuous decay of spray width with increasing ambient pressure. With the relatively lower ambient pressure (4kPa-20kPa), an evident expansion zone is detected as the spray exits from the counter bore. As noted from the experimental pictures, the expansion zone is composed of the central high density liquid core and the peripheral lighter vaporized fuel. The simulation results have presented a perfect resemblance with experimental observations in term of the spray shape and spray angle. To be more specific, the spray width and spray angle both present a trend of decaying with higher ambient pressure in experimental observations. The diminishing of vapor amount can be predicted from the continuing darkening spray zone. In contrast, no clear boundary between liquid and vapor is detected in the liquid mass distribution because of the utilization of diffused interface model in the simulation. Nevertheless, the boundary of fuel is clearly discernible. Moreover, the continuing extending liquid core along with increasing ambient pressure is well captured in the simulations. Until the initial liquid is no longer superheated $(>200 \mathrm{kPa})$, the spray width and angle are stabilized albeit the ambient pressure increases. Overall, the simulation results agree well with experiments qualitatively. More quantitative studies can be found in our previous study[21].
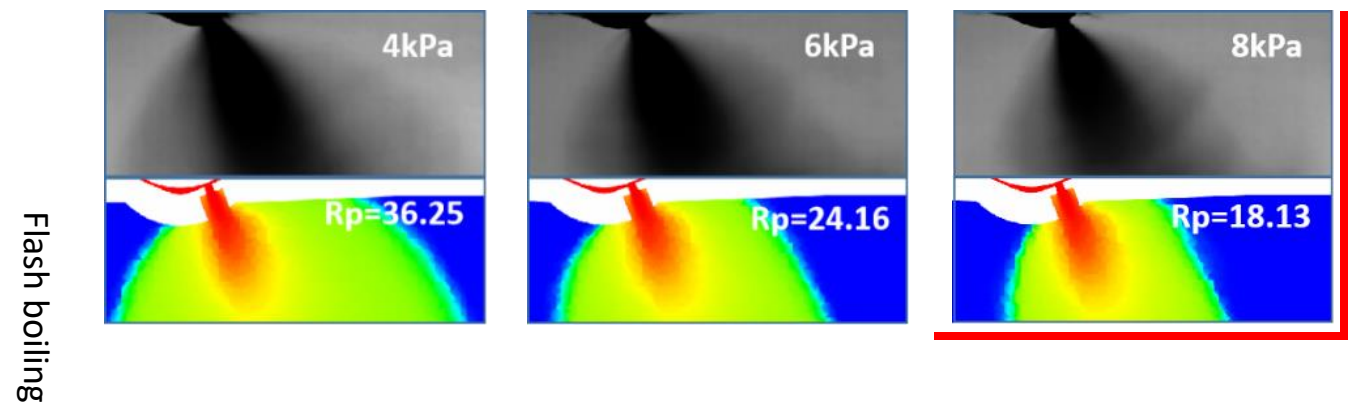


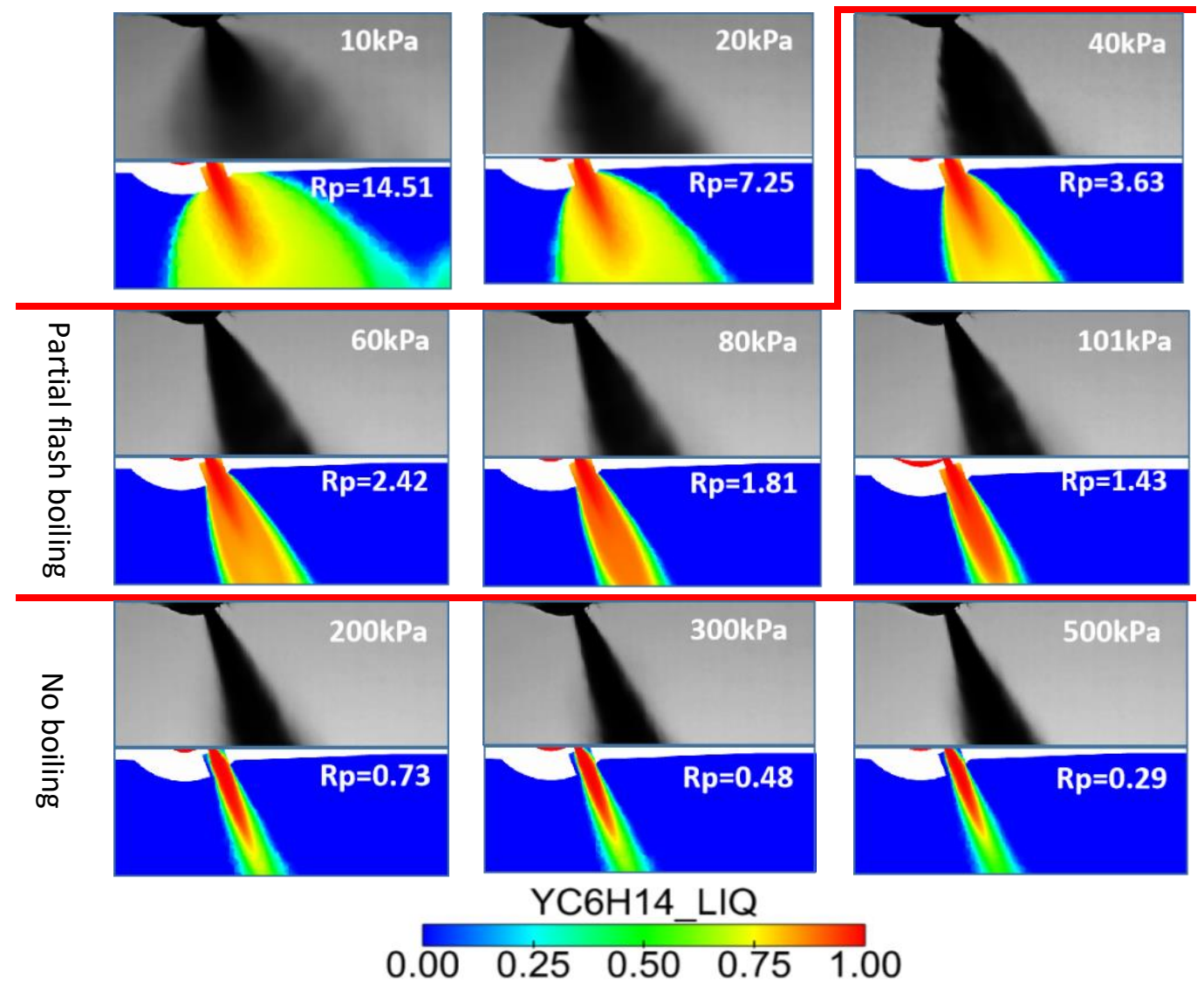

Figure 3 Comparisons between experimental observation and simulation results. The images marked with pressure value and the initial $R p_{\text {ini }}$. represent the experimental and simulation results of the same case respectively.

\subsection{The evolution of superheated region and supersonic fluid}

\subsubsection{Discussion of the internal/external flash boiling}

In this section, we define the dynamic pressure ratio index $R p_{d}$ to indicate the potential existence of phase change or superheated fluid. Distinct with the initial definition, $R p_{d}$ is formulated as the ratio between the saturation pressure under the real-time temperature instead of the initial reservoir fuel temperature and real-time pressure. Thus, potential phase change or superheated region is anticipated if $R p_{d}$ value is above one in the spray region. If the superheated region exists inside the nozzle, it may represent the occurrence of the internal flash boiling. In contrast, if these superheated regions are existed only in the downstream spray region outside the nozzle, the occurred phase change may denote the existence of the external flash boiling.

To detect the exact location and value of the potential phase change or superheated region, the contour of the $R p_{d}$ is illustrated in the following images (left column). In addition, the distribution of Mach number (middle column) and mass fraction of vapor at the initial injection time of $50 \mu \mathrm{s}$ (right column) are presented as well. The isolines appear in the image of the Mach distribution denotes the Mach number equalling one. To improve the readability of each figure, the images have been demonstrated separately based on distinct flash boiling regime. Since there is no precise definition or threshold concerning various flash boiling regimes, the differentiation among different regimes is therefore based on experience. In current work, the flash boiling regime is determined as there exists obvious Mach disk in the spray region $\left(P_{a m b .}=4-20 \mathrm{kPa}\right)$. Several evident characteristics can be concluded for this regime: 1$)$, the jet is instantly expanded as exiting from the counter bore without obvious delaying. 2), the superheated region spans from the inner hole to the downstream spray region as detected 
with the evolution of $R p_{d}$. 3), Mach disk appears in the location with the most intensive phase transition or highest superheated degree. The first two characteristics can forcefully determine this scenario as the internal flash boiling regime according to the initial definition as mentioned in Introduction section. The intensity of evaporation and width of Mach disk continue shrinking with increasing ambient pressure. The other interesting fact is that the supersonic zone generally commences at the end of the liquid core outside the counter bore albeit with the existence of the internal phase change as discussed in previous section. The generated shock wave dimension in the axial direction is less sensitive to the ambient pressure compared to the radial dimension.
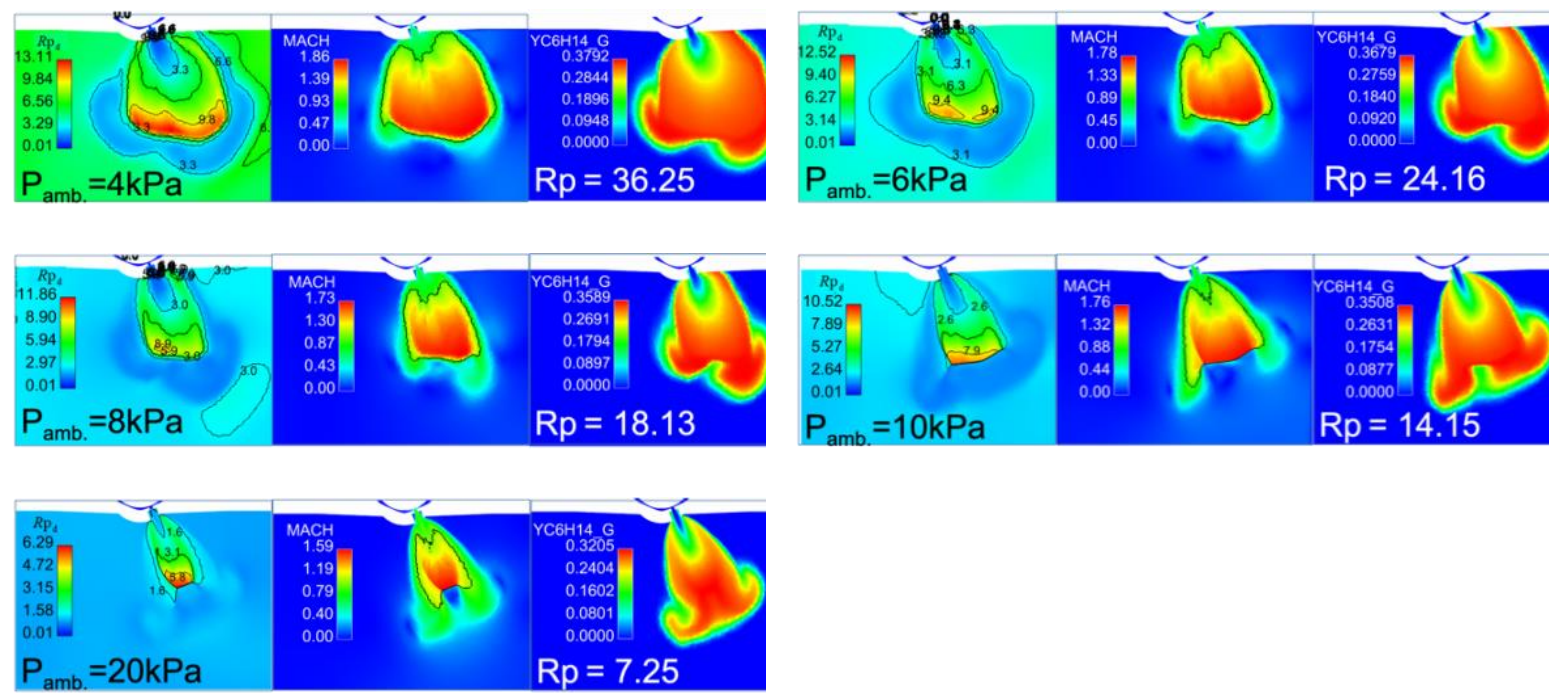

Figure 4 Demonstration of the cases with flash boiling (internal flash boiling), the contours represent real-time phase change index $\left(R p_{d}\right.$, left column), sub/supersonic region (Mach number, middle column) and mass fraction of gaseous fuel ( $Y_{\mathrm{C}_{6} \mathrm{H}_{14} \mathrm{G}}$, right column) at the time instant of $50 \mu \mathrm{s}$ respectively. The isolines in the image of the middle column denote the Mach number equalling one.

As the ambient pressure keeps surging to $40 \mathrm{kPa}$, the Mach disk is not visible anymore (Figure 5). With such high ambient pressure, the bubble growth or nucleation process is strongly inhibited which may be explained by the decrease and decaying of the superheated region $\left(R p_{d}\right)$ in the spray. In this scenario, the fast shattering or expansion process of the jet as exiting from the nozzle is not witnessed any more. Nevertheless, internal slight phase transition behavior is still detectable. Hence this scenario may correspond to the external flash boiling at the low superheated degree as shown in Figure 1. In this regime, the supersonic region in the spray is also shrinking with higher ambient pressure. Moreover, the generated vapor amount is significantly reduced compared to flashing scheme. According to current research, the transition from previous internal flash boiling regime to external flash regime is decided more by the superheated state of the fuel or the expansion of the spray. More quantitative analysis relating to the transition point is presented in following section. The studies here confirm that in the external flash boiling regime, the fluid has been already atomized and entered the two-phase flow regime when exiting from the nozzle and stay in the idling state for some time before starting to expand.
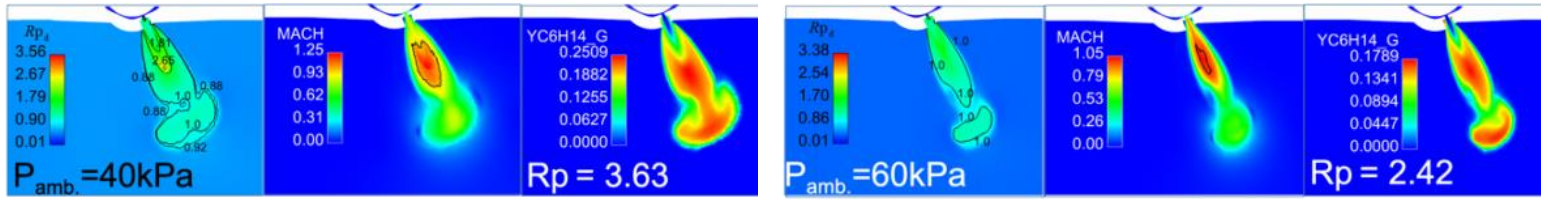

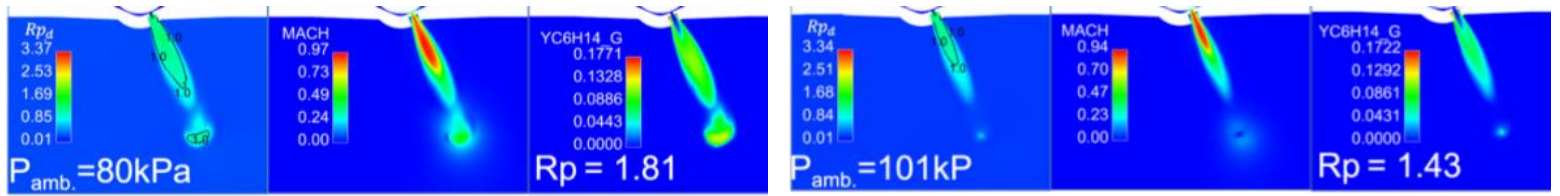

Figure 5 Demonstration of the cases with partial flash boiling, the contours represent real-time phase change index $\left(R p_{d}\right.$, left column), sub/supersonic region (Mach number, middle column) and mass fraction of gaseous fuel $\left(Y_{\mathrm{C} 6 \mathrm{H} 14_{-} \mathrm{G}}\right.$, right column) at the time instant of $50 \mu \mathrm{s}$ respectively. The isolines in the image of the middle column denote the Mach number equalling one.

While the ambient pressure is above $101 \mathrm{kPa}$, the external flash boiling zone or superheated zone has rapidly diminished to the counter bore (See $P_{a m b}=200 \mathrm{kPa}$ case). And the visible phase change zone or superheated region is shrinking to the edge of internal nozzle namely the cavitation region as shown in Figure 6 . On the other hand, the dynamic superheated degree has been decaying with the increment of ambient pressure from initial peak value $R p_{d}=13.1$ to 3.4. The minimal $R p_{d}$ value is located exactly in the upper edge of the inter nozzle, corresponding to the cavitation region. The intensity of evaporation correlates well with $R p_{d}$ value which is proved by the larger mass fraction of vapor $\left(Y_{\mathrm{C} 6 \mathrm{H} 14_{-} \mathrm{G}}\right)$ at higher $R p_{d}$. The amount of vapor has decreased gradually from $38 \%$ to $1 \%$ as the back pressure climbs from $4 \mathrm{kPa}$ to $500 \mathrm{kPa}$. Since no evaporation appears in this situation, the shape of the jet is basically stabilized. As the initial fuel is at subcooled state, only the cavitation physics prevails in the inner hole (see $P_{a m b}=200 \mathrm{kPa}, 500 \mathrm{kPa}$ case). It is reasonable that there is no vapor phase generated in the spray considering the ambient pressure far exceeds the saturation pressure. This phenomenon was mentioned by Oza [22] in the experimental studies of flashing injection. According to Oza, even though the ambient pressure is above the saturation pressure, there still exists two-phase region inside the nozzle which perfectly validates the current simulation.
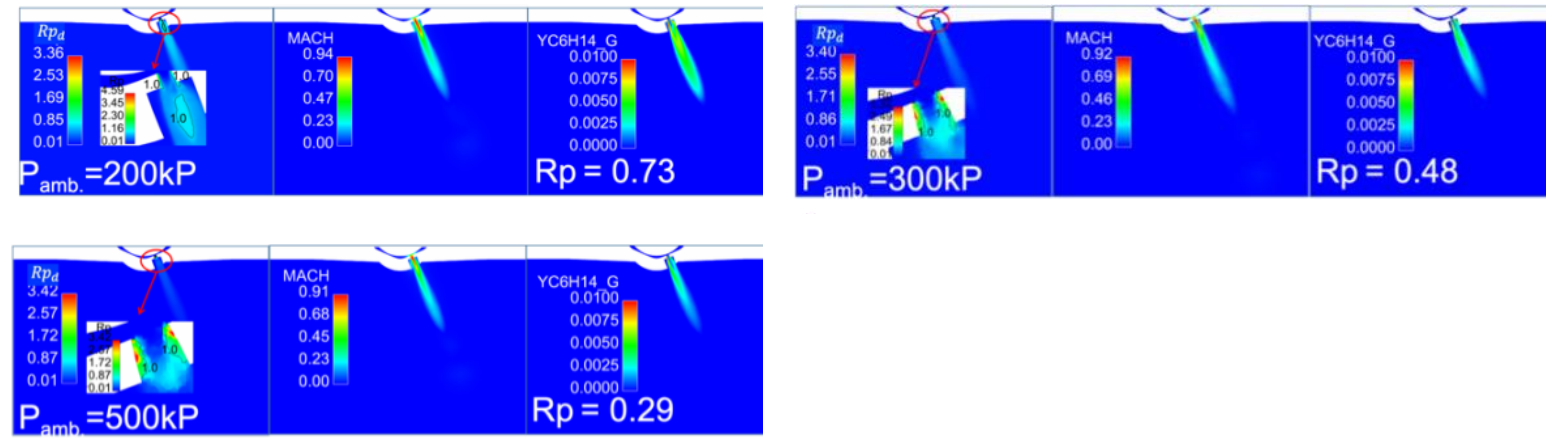

Figure 6 Demonstration of the cases without flash boiling, the contours represent real-time phase change index

$\left(R p_{d}\right.$, left column), sub/supersonic region (Mach number, middle column) and mass fraction of gaseous fuel $\left(Y_{\mathrm{C} 6 \mathrm{H} 14-G}\right.$, right column) at the time instant of $50 \mu \mathrm{s}$ respectively. The isolines in the image of the middle column denote the Mach number equalling one.

\subsubsection{Quantitative analysis of the transition point}

With the gradual increase of the ambient pressure, the flashing regime has been through the transition of the flashing state to partial flashing state and to the final no flashing state. Meanwhile, the transition of intensive flash boiling to slight flash boiling corresponds to the transition of internal flash regime to external flash regime as well. To further clarify this transition point, the variation of Mach number along the central axis from the reservoir to the downstream region is plotted in Figure 7Error! Reference source not found. for each case. Meanwhile, to facilitate the observation of the transition point, the maximum gradient value of Mach number along the axis distance for the ambient pressure of $4 \mathrm{kPa}$ to $40 \mathrm{kPa}$ cases is also marked in the figure. As discussed above, one salient difference between the internal flash boiling and external flash boiling 
lies in the existence of Mach disk. The location of Mach disk generally marks an enormous gradient value of Mach number. As shown in Figure 7, the disappearance of Mach disk within the range of ambient pressure $(20 \mathrm{kPa}-30 \mathrm{kPa})$ has witnessed a significant jump of the variation of maximum Mach number gradient. This can forcefully prove there exists a transition point somewhere between $20 \mathrm{kPa}$ and $30 \mathrm{kPa}$ which corresponds to the range of an initial superheated degree $R p_{\text {ini }}$. 7.25 and 4.83 respectively.

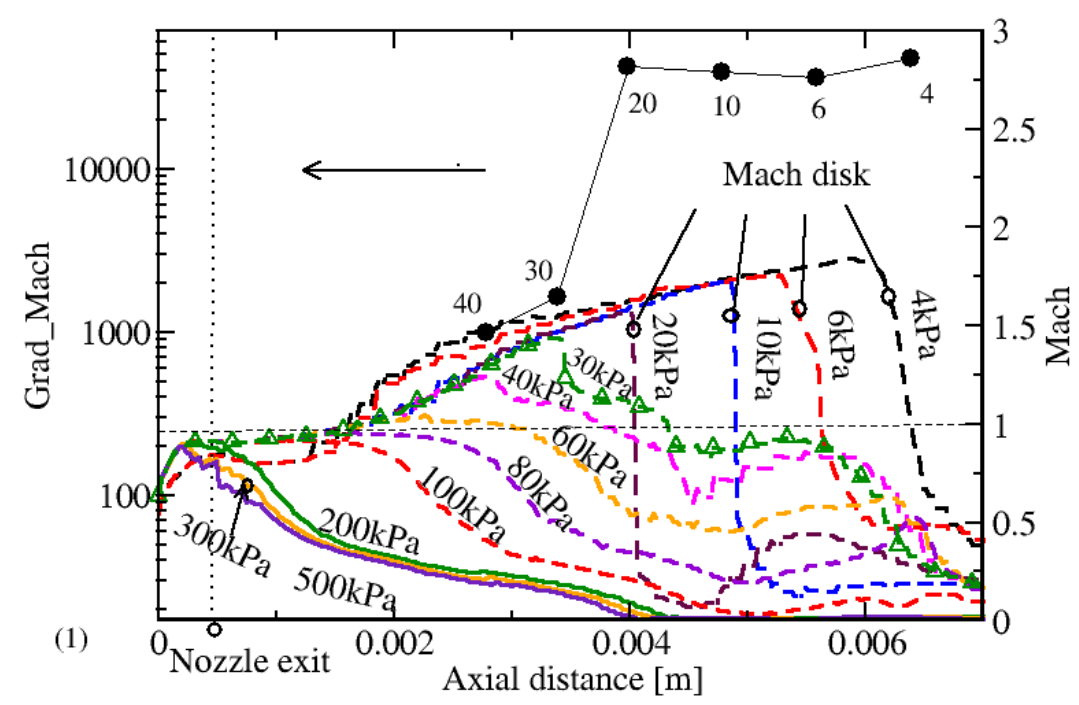

Figure 7 Illustration of Mach number along the central axis from the reservoir to the ambient and the maximum value of gradient with respect to the Mach number along the axial direction under the ambient pressure of $4 \mathrm{kPa}$ $40 \mathrm{kPa}$.

\section{Conclusion}

The study has adopted the numerical simulation strategy to investigate the internal and external flow in flash boiling sprays. By adjusting the ambient pressure, the spray has been through the transition of non-flash boiling to flash boiling continuously in which the transition regime from external flash boiling to internal flash boiling is also detected. A good qualitative agreement is achieved as comparing the simulation with experimental results. A dynamic pressure ratio $\left(R p_{d}\right)$ based on the real-time temperature and pressure is defined to present the evolution of the potential phase change and superheated region in the fluid. The potential transition points between different flashing regimes are discussed as well based on current simulation results. Several conclusions have been drawn from this study:

1) The fluid inside the nozzle is situated at two-phase state irrespective of the initial superheat degree.

2) Internal flash boiling exists in the scenarios with initial high superheated fluid and clear Mach disk is visible in this regime.

3) Within the sole variable system as of ambient pressure, the regions for external flash boiling and expansion has been diminishing with increasing ambient pressure.

4) The transition from the flashing regime to slight-flashing regime may correspond to the change of internal flash boiling to external flash boiling regime.

\section{Acknowledgement}

The work is financially supported by National Science Foundation of China under Grant NO. 52076119. 


\section{References}

[1] M. Mojtabi, G. Wigley, and J. Hélie, "The effect of flash boiling on the atomization performance of gasoline direct injection multistream injectors," At. Sprays, vol. 24, no. 6, pp. 467-493, 2014.

[2] D. Kawano, Y. Goto, M. Odaka, and J. Senda, "Modeling atomization and vaporization processes of flash-boiling spray," SAE Tech. Pap., vol. 2004, no. 724, 2004.

[3] R. D. Oza and J. F. Sinnamon, "An experimental and analytical study of flash-boiling fuel injection," SAE Tech. Pap., vol. 830590, 1983.

[4] V. Cleary, P. Bowen, and H. Witlox, "Flashing liquid jets and two-phase droplet dispersion. I. Experiments for derivation of droplet atomisation correlations," J. Hazard. Mater., vol. 142, no. 3, pp. 786-796, 2007.

[5] H. Guo, B. Wang, Y. Li, H. Xu, and Z. Wu, "Characterizing external flashing jet from single-hole GDI injector," Int. J. Heat Mass Transf., vol. 121, pp. 924-932, 2018.

[6] T. C. Lin, Y. J. Shen, and M. R. Wang, "Effects of superheat on characteristics of flashing spray and snow particles produced by expanding liquid carbon dioxide," J. Aerosol Sci., vol. 61, pp. 27-35, 2013.

[7] G. Lamanna, H. Kamoun, B. Weigand, and J. Steelant, "Towards a unified treatment of fully flashing sprays," Int. J. Multiph. Flow, vol. 58, pp. 168-184, 2014.

[8] R. D. Reitz, "A photographic study of flash-boiling atomization," Aerosol Sci. Technol., vol. 12, no. 3, pp. 561-569, 1990.

[9] S. Shen, Z. Che, T. Wang, M. Jia, and K. Sun, "Numerical Study on Flash Boiling Spray of Multi-Hole Injector," SAE Int. J. Fuels Lubr., vol. 10, no. 2, 2017.

[10] H. Guo, Y. Li, H. Xu, S. Shuai, and H. and Zhang, "Interaction between underexpanded flashing jets: A numerical study," Int. J. Heat Mass Transf., vol. 137, pp. 990-1000, 2019.

[11] J. Lacey et al., "Generalizing the behavior of flash-boiling, plume interaction and spray collapse for multi-hole, direct injection," Fuel, vol. 200, pp. 345-356, 2017.

[12] D. Paredi, T. Lucchini, G. D’Errico, A. Onorati, L. Pickett, and J. Lacey, "Validation of a comprehensive computational fluid dynamics methodology to predict the direct injection process of gasoline sprays using Spray G experimental data," Int. J. Engine Res., vol. 21, no. 1, pp. 199-216, 2020.

[13] C. Price, A. Hamzehloo, P. Aleiferis, and D. Richardson, "Numerical modelling of fuel spray formation and collapse from multi-hole injectors under flash-boiling conditions," Fuel, vol. 221, no. August 2017, pp. 518-541, 2018.

[14] O. Redlich and J. Kwong, "On the thermodynamics of solutions; an equation of state; fugacities of gaseous solutions," Chem. Rev., vol. 44, no. 1, p. 233, 1949.

[15] P. J. Linstrom and W. G. Mallard, "The NIST Chemistry WebBook: A chemical data resource on the Internet," J. Chem. Eng. Data, vol. 46, no. 5, pp. 1059-1063, 2001.

[16] S. Yang, P. Yi, and C. Habchi, "Real-fluid injection modelling and LES simulation of the ECN Spray A injector using a fully compressible two-phase flow approach," Int. J. Multiph. Flow, p. 103145, 2019.

[17] H. Guo, Y. Li, B. Wang, H. Zhang, and H. Xu, "Numerical investigation on flashing jet behaviors of single-hole GDI injector," Int. J. Heat Mass Transf., vol. 130, pp. 50-59, 2019.

[18] Z. Bilicki and J. Kestin, "Physical aspects of the relaxation model in two-phase flow," Proc. R. Soc. Lond. A, vol. 428, no. 1875, pp. 379-397, 1990.

[19] P. Downar-Zapolski, Z. Bilicki, L. Bolle, and J. Franco, "The non-equilibrium relaxation model for one-dimensional flashing liquid flow," Int. J. Multiph. Flow, vol. 22, no. 3, pp. 473-483, 1996.

[20] P. K. Senecal, J. K. Richards, E. Pomraning, and E. Al., "A New Parallel Cut-Cell Cartesian CFD Code for Rapid Grid Generation Applied to In-Cylinder Diesel Engine Simulations," SAE Tech. Pap., 2007.

[21] H. Guo, L. Nocivelli, R. Torelli, and S. Som, "Towards understanding the development 
ICLASS 2021, 15 th Triennial International Conference on Liquid Atomization and Spray Systems, Edinburgh, UK, 29 Aug. - 2 Sept. 2021 and characteristics of under-expanded flash boiling jets," Int. J. Multiph. Flow, vol. 129, p. 103315, 2020.

[22] R.D.Oza, "On the Mechanism of Flashing Injection of Initially Subcooled Fuels," J. Fluids Eng., vol. 106, pp. 105-109, 1984. 